Revue Sciences/Lettres

Sciences / Lettres

$4 \mid 2016$

Baba Yaga en chair et en os

\title{
Sorcière ou nourricière : la Baba Yaga à l'épreuve de la pensée psychanalytique
}

Cécile Rousselet

\section{(2) OpenEdition \\ Journals}

Édition électronique

URL : https://journals.openedition.org/rsl/967

DOI : $10.4000 / \mathrm{rsl} .967$

ISSN : 2271-6246

Éditeur

Éditions Rue d'Ulm

\section{Référence électronique}

Cécile Rousselet, « Sorcière ou nourricière : la Baba Yaga à l'épreuve

de la pensée psychanalytique », Revue Sciences/Lettres [En ligne], 4 | 2016, mis en ligne le 16 janvier

2016, consulté le 21 septembre 2021. URL : http://journals.openedition.org/rsl/967 ; DOI : https://

doi.org/10.4000/rsl.967

Ce document a été généré automatiquement le 21 septembre 2021.

(c) Revue Sciences/Lettres 


\title{
Sorcière ou nourricière : la Baba Yaga à l'épreuve de la pensée psychanalytique
}

\author{
Cécile Rousselet
}

1 « Mère nourricière - mère sorcière ». Ces deux expressions peuvent sembler antagonistes mais révèlent en réalité, dans leur conjonction, l'essence même de la représentation maternelle dans les contes russes. À première lecture, la mère nourricière apparaît comme une femme totalement bonne envers son enfant, la marâtre ou la Baba Yaga, son prolongement, comme des personnages cruels et parfois même cannibales, dévorant les enfants comme des " poulets ${ }^{1}$ ». Pourtant, les Baba Yaga apparaissent rapidement comme des personnages véritablement ambigus, distribuant leurs pouvoirs destructeurs ou bienfaiteurs au gré des contes et des sentiers. C'est dans cette vision complexe et plurielle des pouvoirs des sorcières que l'approche psychanalytique trouve sa place dans le champ d'études des contes d'Afanassiev. Une telle approche se fonde d'emblée sur la question du fantasme : toute analyse psychanalytique des contes en est une analyse "pragmatique ». Les Baba Yaga sont des supports de fantasmes d'agressivité et de destructivité, au sein d'un mécanisme inconscient collectif ${ }^{2}$. Elles ne peuvent dès lors tenir un rôle unique, puisqu'elles endossent tour à tour ceux que les interlocuteurs de ces contes veulent leur faire porter. Nous appelons ces interlocuteurs les « acteurs pragmatiques » du conte, en ce sens qu'ils participent, en les écrivant, lisant et racontant, à faire vivre la composante fantasmatique des textes. Ces sorcières, que l'on pouvait considérer comme des personnages stéréotypés, se manifestent ici dans toute leur complexité. Nous mettrons deux contes issus du recueil d'Afanassiev, "Vassilissa la Belle ${ }^{3}$ " et " Tomassounet $^{4}$ " à l'épreuve de théories psychanalytiques, celles de Sigmund Freud (sur la transposition pulsionnelle et l'étayage des pulsions dans la libido infantile), Melanie Klein (dans son analyse des imagos maternelles et de la position schizo-paranoïde du jeune enfant), mais aussi les analyses proposées par Jean Bellemin-Noël, Marthe Robert, ou Nicole Belmont sur les contes et leurs fantasmes. 
2 Néanmoins, une telle approche psychanalytique est elle-même mise à l'épreuve par la complexité du matériau sur lequel elle se penche. Tout d'abord, les contes sont des structures narratives établies ${ }^{5}$. Le conte a ses codes, et un pôle agressif inévitable dans l'économie narrative, économie que l'analyse pragmatique des Baba Yaga ne peut ignorer. En outre, les contes d'Afanassiev sont des textes littéraires : il ne faut pas tomber dans le piège d'un certain essentialisme, qu'une lecture psychanalytique, d'une certaine manière, encourage. Une analyse pragmatique psychanalytique des contes russes s'inscrit dès lors davantage dans l'étude des processus inconscients qui sous-tendent la narration - le genre féminin de la Baba Yaga dans des contes réécrits par un auteur masculin qu'est Afanassiev, fait de ce personnage par exemple un lieu d'investissement fantasmatique sexuel - que dans une démarche illustrative de conceptualisations psychanalytiques figées. Le conte littéraire fait sien les investissements pulsionnels qui structurent les textes et la Baba Yaga se place au cœur d'une circulation fantasmatique et poétique, dans une négociation permanente entre processus imaginaire et réécriture littéraire.

\section{La Baba Yaga : un chaudron pulsionnel. Trois entrées par la psychanalyse}

3 L'analyse psychanalytique des personnages de Baba Yaga dans les contes populaires russes peut se faire selon trois voies. Une première entrée par la psychanalyse consiste en l'analyse du motif de l'avalement. Dans la dynamique fantasmatique du conte, avaler représente un retour à la fois rassurant ou menaçant au sein maternel, une symbiose la plupart du temps mortifère. Jean Bellemin-Noël, dans Les Contes et leurs fantasmes, écrit :

Créer une dépendance jusqu'à vouloir dévorer, jusqu'à vouloir récupérer l'autre intégralement. Nourrir pour avoir de quoi se nourrir. Dévorer pour ne pas risquer de perdre, de voir l'autre nous échapper 6

Avaler ses enfants, c'est donc les réintégrer, pendant négatif et thanatique de l'acte érotique qu'est de donner naissance. Si l'on reprend cette théorie, il s'agit donc ici, dans la dévoration par la Baba Yaga, de l'agressivité maternelle que l'enfant ressentirait et fantasmerait dans le conte. Néanmoins, les manifestations pulsionnelles à l'œuvre dans la formation de l'objet conte sont complexes, et il est impossible alors de considérer «la sorcière cannibale » de manière essentielle, ou même de pouvoir interpréter de manière univoque le comportement d'une sorcière précise dans un conte précis. La pluralité de significations que chaque acte cannibale peut offrir nous invite à nous demander si la thèse selon laquelle chaque sorcière transpose, sur le plan imaginaire, le comportement hostile d'une mère envers son enfant dans la réalité, puis sa transformation inconsciente, est la seule donnée explicative à l'analyse du comportement cannibale dans nos contes. Nicole Belmont présente à plusieurs reprises les textes comme des lieux d'inversion fantasmatique : l'enfant qui souhaite plus que tout réintégrer sa mère, établir une fusion avec elle, qu'aucune frustration ne pourrait ternir, est un enfant « cannibale » lui-même. La sorcière, menaçant de le dévorer, n'est donc que le pendant de son agressivité. Géza Roheim, dans son introduction à Psychoanalysis and anthropology, décrit le phénomène suivant :

Freud has interpreted the phantasy of the father as an animal that bites (wolf) and of being eaten by the father as the manifestation of the negative Oedipus complex of passivity in relationship to the father. But this phantasy is also a sequel to the 
tale of the cannibal mother who, in turn, as we saw above, is the "cannibal" child in reverse $^{7}$.

5 La position dévorante de la sorcière semble donc davantage être l'impression fantasmatique de l'agressivité de l'enfant lui-même envers sa mère, recyclée dans le conte par un déplacement sur le personnage de la sorcière, ce déplacement opérant ainsi une épargne psychique pour l'enfant : il s'agit là d'une deuxième entrée dans le conte par la psychanalyse. Celui-ci est traversé de tensions infantiles, fixé à la fois à un stade narcissique - il projette donc sa libido sexuelle sur lui-même, ou ses substituts identificatoires - et satisfaisant ses pulsions sexuelles partielles. Il manifeste alors envers lui-même une grande agressivité sexuelle orale, qu'il réinvestit dans l'imaginaire, en la déplaçant donc sur le personnage de la sorcière. Comme l'écrit Nicole Belmont, l'imaginaire fantasmatique des contes se forme par régression à un stade antérieur de la libido, fixé aux pulsions partielles, et notamment, ici, orales ${ }^{8}$. Ainsi, les sorcières deviennent les manifestations, sur le mode imaginaire, de pulsions inconscientes archaïques, infantiles, fixées au stade oral. Refoulées, les angoisses de dévoration sont recyclées dans la vie fantasmatique, et donc dans l'imaginaire des contes. La formation des Baba Yaga relève dès lors de la constitution d'une imago maternelle destructrice, au sens kleinien. Selon Melanie Klein ${ }^{9}$, l'enfant vit, au début de sa vie, une position schizoparanoïde dans laquelle il n'y a pas d'ambivalence. Le jeune enfant introjecte son environnement, selon deux modalités : amour et haine. De là, il se crée des imagos, qui sont des représentations psychisées de ce qu'il éprouve face au monde : il y a donc des imagos positives et des imagos négatives. Melanie Klein ajoute que ces imagos sont étroitement liées au vécu que l'enfant éprouve par rapport à sa mère : le sein qui comble et qui nourrit devient le «bon sein ", imago positive ; tandis que le sein qui frustre, qui se refuse, qui se sépare, est le «mauvais sein », imago détestable. La création des Baba Yaga relève de la formation fantasmatique d'une représentation du "mauvais sein » dans le conte. La sorcière est donc un fantasme compensatoire, toute l'activité sexuelle orale que l'enfant éprouve envers sa mère étant recyclée dans l'imaginaire selon un scénario qui serait : «Je veux te manger, donc tu me manges. »

6 Une troisième voie d'accès psychanalytique au personnage de la Baba Yaga concerne la difficile sexualisation de la mère par l'enfant, notamment au moment de l'CEdipe, qui se retrouve alors condensée, tout comme le rêve condense les pulsions refoulées en les voilant, dans le personnage de la Baba Yaga. Selon Marthe Robert, ce sont avant tout les personnages masculins idéalisés, dans les œuvres qu'elle étudie, qui sont des avatars du père tout puissant et fantasmé de l'đEdipe et du roman familial que l'enfant se construit au cours de sa petite enfance ${ }^{10}$. Mais les personnages féminins des contes de fées, et notamment les sorcières, sont tout autant des avatars de ce « roman familial ». Au cours de celui-ci, selon Marthe Robert, l'enfant ne cesse de manifester une certaine agressivité envers ses parents puisqu'ils lui mentent, selon son fantasme. Figure fantasmatique, la sorcière prend en charge, donc, cette agressivité, au nom d'une épargne psychique. D'autre part, Sigmund Freud, dans " Le roman familial des névrosés ${ }^{11}$ » insiste sur le fait que, selon lui, le stade de l'enfant bâtard est un stade sexuel, contrairement au premier stade de l'enfant trouvé, puisque l'enfant sexualise sa mère en lui prêtant des amants, dont le plus illustre est son géniteur. Cette sexualisation de la mère peut être vécue, par l'enfant, comme une violence, car nouvelle ; elle est une tension qui vient bouleverser son équilibre psychique. L'agressivité qu'il ressent alors est à la fois dirigée contre sa mère, sexualisée trop brutalement, et envers lui-même, ce lui-même sexualisant sa propre 
mère. L'enfant, inconsciemment, est dédoublé, et soumis aux affres de la découverte de la sexualité. Sa libido se fixe peu à peu à sa mère - avant le stade œdipien, déjà - et génère alors des angoisses infantiles. En effet, pour l'enfant, si sa mère est sexualisée, elle est aussi détentrice des pouvoirs de la sexualité, et par là, elle est en mesure de castrer son enfant. Ce sont ces angoisses que l'enfant ressent qui peuvent être relayées, sur le plan imaginaire, par la création de femmes violentes aux pouvoirs oraux - donc sexuels - forts.

L'appareil théorique psychanalytique a ainsi une réelle pertinence dans l'interprétation des contes et du personnage de la Baba Yaga. Mais en tant que personnage littéraire des Contes d'Afanassiev, la Baba Yaga est un personnage produit dans une certaine mesure par un imaginaire collectif, mais, doté de spécificités esthétiques et poétiques, dépasse le cadre d'interprétation psychanalytique, ou du moins le nuance.

\section{Les théories psychanalytiques à l'épreuve du personnage littéraire de la Baba Yaga}

8 La littérarisation de la Baba Yaga met d'emblée en valeur la dimension fantasmatique mortifère de la sorcière. Vladimir Propp soulignait la cécité de la Yaga, comme lien à l'invisible et à la mort, cette mort que l'on retrouve dans la description de l'isba de Baba Yaga dans « Vassilissa la Belle ». C'est aussi dans l'outrance de la description des pouvoirs oraux qu'Afanassiev surcharge le pôle mortifère et oriente la narration autour du personnage de la sorcière. Dans « Vassilissa la Belle », l'auteur théâtralise la Baba Yaga, tant dans son agressivité - celle-ci s'écrie « Pouah, pouah, cela sent la chair russe ${ }^{12}$ !» -, que dans la description même qu'il fait de sa maison:

Toute la nuit et tout le jour étaient passés, ce n'est qu'au soir qu'elle atteignit la clairière où était l'isba de la baba Yaga: elle était entourée d'une palissade faite d'ossements humains, plantés de crânes humains dont les yeux luisaient; au portail, des jambes étaient placés en guise de traverse, des bras servaient de verrous ; une bouche aux dents aiguës tenait lieu de serrure ${ }^{13}$.

Afanassiev insiste aussi sur l'outrance de son personnage en mettant en scène de manière euphorique la satisfaction libidinale sexuelle que lui apporte la dévoration orale, comme dans le conte « Tomassounet » :

Avale-tout accourut, d'un bond elle entra dans l'isba, s'empiffra, ressortit et se mit à se rouler dans l'herbe en chantant: "Je me couche, je me roule, bien gavée de Tomassounet! $»^{14}$

Et pourtant, tout en étant un personnage "plein », presque saturé esthétiquement, la Baba Yaga est aussi, par certains aspects, un personnage troué, aux béances explicites. Les descriptions physiques sont incomplètes, et la narration est soumise à des vides diégétiques. Le texte ne dit pas, par exemple, ce que la Yaga fait de ses journées dans «Vassilissa la Belle »: c'est par ces trous que le texte offre un espace d'investissement fantasmatique. Ces brèches sont lieux de création, d'élaboration imaginaire personnelle. La Baba Yaga s'impose comme présence charnelle forte et puissante, mais se dérobe, laissant place alors à un vide diégétique qu'il incombe à chacun de combler et de poétiser.

11 La Baba Yaga est tendue entre incarnation, souvent comique, et tension vers une existence plus immémoriale. Elle ronfle dans "Vassilissa la Belle », et dévore le tronc de l'arbre sur lequel s'est réfugié Tomassounet dans le conte du même nom, dents en avant ; 
mais en même temps elle est un gouffre qui semble toujours laisser imaginer des profondeurs que le texte ne décrit pas. La Baba Yaga se trouve dès lors, dans le texte, au centre d'un processus dialogique : personnage effrayant mais dont l'effroi est distancié dans le texte par ses traits comiques et grotesques, elle s'inscrit dans un écart entre l'investissement fantasmatique qu'elle organise et la trivialité de sa textualisation.

En outre, c'est aussi un personnage familier - avatar maternel - qui est inquiété par son cannibalisme, donc sa capacité à devenir l'envers d'elle-même, à nier son rôle maternel, dans un effet poétique d' "inquiétante étrangeté ». Il y a double distance au sein du personnage, et par ce double mouvement distantiel, la Baba Yaga s'inscrit textuellement comme ambivalente. Elle est dans un entre-deux, entre incarnation comique et tension vers un ailleurs, entre personnage avatar d'une mère réelle et fantasme d'une imago agressive toute puissante. C'est à cause de cet entre-deux qu'il semble nécessaire de relativiser l'approche kleinienne de la Baba Yaga.

13 La Baba Yaga est dans un entre-deux dans le sens où elle semble menacer de dévorer pour mieux ne pas dévorer. La sorcière représente, dans le conte, un pôle mortifère pour mieux pointer du doigt la véritable menace orale, qui est celle du personnage que l'on considérait comme la "bonne mère ». La Baba Yaga devient alors une mère «suffisamment bonne » au sens de Winnicott ${ }^{15}$, dans l'économie narrative du conte : elle présente la menace orale opérée par la vraie mère, pour mieux permettre au héros de s'en détacher.

14 À cet égard, le conte "Vassilissa la Belle » est particulièrement significatif puisque les rôles de bonne mère et figure maternelle dévorante sont inversés. La poupée représente la permanence du lien qui unit Vassilissa à sa véritable mère, présentée comme bienfaitrice. Néanmoins, si cette poupée aide la jeune fille à accomplir les tâches que lui imposent sa marâtre et ses sœurs, c'est en échange de nourriture. La poupée, en mangeant pour secourir la jeune fille, est une image de la menace orale dont Vassilissa pourrait être victime si elle ne rompt pas le lien fusionnel à sa mère morte. Au contraire, la Baba Yaga, ne mangera la jeune fille que si elle ne travaille pas assez: "Tu vas travailler chez moi quelque temps; si je suis contente, je te donnerai du feu, sinon je te mangerai ${ }^{16}$. $\gg$ La sorcière répète la menace orale que la poupée exprime, et propose à Vassilissa de s'en dégager : son ambiguité réside dans le fait que tout en menaçant de dévorer la jeune fille, elle est aussi agent de son développement et de son autonomisation. $\mathrm{Au}$ fur et à mesure de son séjour chez la Baba Yaga, Vassilissa doit travailler, de plus en plus : elle prépare le repas, puis elle doit tisser elle-même le fil grâce au métier à tisser que lui a confectionné sa poupée - et lors de cette dernière étape, la poupée ne mange pas -, avant d'aider la jeune fille. Enfin, au moment de coudre les douze chemises, Vassilissa opère seule : elle est autonome. La poupée reste dans la poche de la jeune fille et n'en sort plus : intégrée à la personnalité de sa propriétaire, elle laisse celle-ci réussir par ses propres moyens.

Dans "Tomassounet », ce rôle initiatique de la Baba Yaga quant à la question orale est également explicite. Au début du conte, le garçon va régulièrement pêcher seul, et le symbolisme de ces voyages semble indiquer son besoin d'émancipation. Néanmoins, sa mère le rappelle à la rive pour lui donner du lait et du fromage blanc, deux aliments qui symbolisent le lien fusionnel procuré par l'allaitement. Ainsi, de la mère nourricière à la mère qui gave son enfant, le pas est vite franchi : la mère de Tomassounet donne son lait autant qu'elle semble l'imposer au garçon - en obéissant à ses demandes libidinales, mais en l'empêchant de voguer au loin et de s'autonomiser. Il est remarquable de constater que 
lorsque « Avale-tout» tente d'attirer le garçon sur la rive, elle se fait faire une douce voix "toute pareille à celle de la mère »: on voit donc dans cette confusion l'ambiguïté de chacun des pôles maternels. On remarque aussi que "Avale-tout» est associée à un personnage masculin, le forgeron: les deux hommes du conte (le père qui construit à Tomassounet sa barque et le forgeron associé à la Yaga) sont des agents de la castration ces personnages renvoient au père castrateur de la théorie freudienne du complexe d'ÆEdipe, ce père qui, parce qu'il pose l'interdit de l'inceste, dénoue la fusion entre la mère et l'enfant; ces deux figures permettent ainsi au jeune garçon de s'autonomiser en se détachant de la sphère maternelle et orale archaïque. L'évolution du rapport à la nourriture que vit le garçon est d'ailleurs explicite dans la mention des crêpes que Tomassounet mange en revenant chez lui : ce n'est plus du lait brut mais un mets cuisiné. Les contes sont alors un hymne au plaisir de manger, mais de manger bien.

Le personnage littéraire de la Baba Yaga, par sa condensation poétique, peut d'une certaine manière mettre en scène les investissements fantasmatiques qui semblent avoir présidé à son élaboration populaire. Mais le texte littéraire lui donne une plus grande ambivalence, ce qui permet de relativiser les positions psychanalytiques faisant de la sorcière l'incarnation des pulsions archaïques orales et mortifères de l'enfant, tout en les enrichissant.

\section{L'apport de la théorie littéraire pour la compréhension fantasmatique et pragmatique du conte}

Dans la dynamique même de la narration, qui suit le cheminement du héros, la Baba Yaga dérange, vient faire un écart. Son déploiement textuel est tout entier dans cet écart. Il s'agit pour le héros, et pour la trame même du récit, de rétablir l'ordre narratif, et de supprimer la sorcière. L'ambivalence même de ce personnage, sa faculté à toujours se distancier comiquement ou de manière inquiétante, produit déjà un espace de mise à mort potentiel : son incarnation comique ou mortifère est sapée par la narration, et donc possiblement anéantissable. La Baba Yaga s'inscrit dès lors dans un processus carnavalesque $^{17}$ : son outrance est subversive, et la dimension régressive - et par là angoissante - de ses agissements est liquidée à la fin du texte, ce dénouement permettant le retour à un certain ordre (narratif et affectif).

Dans la dynamique du conte, le héros nécessite donc un face-à-face avec la sorcière. Dans ce face-à-face, la destruction de cette mère dévorante est fondamentale: elle souligne l'évacuation du pôle mortifère dans le conte et, à un niveau plus pragmatique de lecture, la liquidation des schèmes archaïques qui organisaient fantasmatiquement le personnage de la Baba Yaga. Les morts des sorcières et marâtres fascinent. Dans "Vassilissa la Belle », on peut lire:

On apporte le feu dans la chambre. Les yeux des crânes se mettent à fixer la marâtre et ses filles et à les brûler vives. Elles se jettent de-ci de-là, mais où qu'elles se fourrent, partout les yeux les suivent. Au matin, il ne restait d'elles qu'un petit tas de cendres dans un coin. Seule, Vassilissa avait été épargnée ${ }^{18}$.

19 Il est un autre cas remarquable : il s'agit du cas où la Baba Yaga meurt de la mort réservée au héros. Dans "Tomassounet », nous considérons que la fille d'Avale-tout est un avatar diégétique de la Yaga elle-même : faire rôtir la fille, c'est une manière de tuer Avale-tout, 
représentation maternelle, tout en déplaçant le meurtre. Mais sur le plan fantasmatique aussi, au nom d'une épargne psychique, il est plus aisé de tuer la fille de la sorcière, puisque tuer véritablement la mère (ou sa représentation directe dans le conte) serait désorganisant psychiquement. La sorcière, donc, en dévorant impunément sa propre fille se dévore elle-même. La sorcière est renvoyée à ses pulsions archaïques, à sa menace orale, et en dégage le héros. Le spectacle de l'anthropophagie maternelle, réintégrant sa propre progéniture, est ainsi salutaire pour l'enfant: s'il manifeste la violence d'une relation fusionnelle de l'enfant à sa mère, il est aussi le vecteur d'une connaissance sur le monde. L'enfant, héros du conte et soumis aux menaces cannibales d'une sorcière, devient alors, par la destruction de sa mauvaise mère, la dynamique fondamentale de la sublimation des déficiences parentales.

Dans un carnaval jouissif, la Baba Yaga est aussi sacrifiée par la narration, et son exécution, racontée de manière complaisante, permet tant au héros qu'aux «acteurs pragmatiques » des contes, d'accéder à un stade supérieur de maturité. En la détruisant, ceux-ci réduisent la personnification de leur fantasme dans le conte à un vague souvenir dont on ne garde guère de traces, sinon celle d'une épreuve, traversée avec succès. La Baba Yaga efface les traces de son passage à l'aide de son balai, le balai tenant alors lieu d'image poétique du refoulement. Nicole Belmont, à propos du «Conte du genévrier » des frères Grimm, indique :

La violence narrative avait un effet cathartique dans la mesure où elle donnait un statut externe à des pulsions inconscientes intenses désireuses de se faire jour. La mise en récit, la narrativisation, permettait en outre une désintrication de ces pulsions, qui s'exprimaient dès lors en une séquence libératrice, depuis l'oralité et le cannibalisme jusqu'à l'acquisition d'une identité sexuée ${ }^{19}$.

21 La plasticité du conte est sans doute ce qui le rend à la fois si riche et si complexe, adaptant les fantasmes à la narration, et influençant par la narration les fantasmes. Dès lors, incarnant les pulsions de mort, invitant au dépassement de ces pulsions archaïques, les sorcières sont fondamentalement ambivalentes, par leur seule présence dans la diégèse. Elles sont le lieu, pour les acteurs pragmatiques des contes, d'un «transfert » de pulsions infantiles et partielles liées à Thanatos, et elles offrent, en acceptant ce transfert puis en étant sacrifiées, la possibilité d'atteindre Eros. Profondément métapoétiques, nos contes mettent en abyme leur fonctionnement pragmatique.

\section{Conclusion}

Nous avons pu dégager l'apport que représente la théorie psychanalytique dans l'approche des contes populaires et notamment de l'étude de la Baba Yaga. Néanmoins, il faut souligner l'importance de faire jouer les théories les unes avec et contre les autres, et surtout de les heurter aux textes. « Nourricière ou sorcière : la Baba Yaga à l'épreuve de la pensée psychanalytique »: il s'agit bien là d'une épreuve, car personnage littéraire, la Baba Yaga a la spécificité d'être un haut lieu fantasmatique des contes, eux-mêmes produits, indéniablement, dans une certaine mesure, par des mouvements collectifs. L'écoute attentive des mécanismes inconscients a permis de dégager l'ambivalence de la Baba Yaga, dans cet entre-deux, entre la sorcière et la bonne nourricière. Mais c'est au cœur du texte que se déploie toute la saveur de cette ambivalence, qui fait encore aujourd'hui de la Baba Yaga un personnage si haut en couleurs. 


\section{BIBLIOGRAPHIE}

Afanassiev, Alexandre Nikolaïevitch, Contes populaires russes, trad. fr. Lise Gruel-Apert, Paris, Imago, 2014.

Bakhtine, Mikhaïl, L'Ëuvre de François Rabelais et la culture populaire au Moyen Âge et sous la Renaissance, trad. Andrée Robel, Paris, Gallimard, 1982.

Bellemin-Noël, Jean, Les Contes et leurs fantasmes, Paris, PUF, 1983, p. 27-29.

Belmont, Nicole, Mythe, conte et enfance : les écritures d'Orphée et de Cendrillon, Paris, L'Harmattan, 2010.

Freud, Sigmund, Le Roman familial du névrosé et autres textes, trad. Danièle Voldman et Olivier Mannoni, Paris, Payot, 2014.

Klein, Melanie, "Le développement précoce de la conscience chez l'enfant », in Essais de psychanalyse, trad. fr. Marguerite Derrida, Paris, Payot, 1968.

Propp, Vladimir Iakovlevich, Morphologie du conte, trad. Claude Ligny, Paris, Gallimard, 1970.

Robert, Marthe, Roman des origines et origines du roman, Paris, Grasset, 1988.

Róheim, Géza, Psychanalyse et anthropologie : Culture - Personnalité - Inconscient, trad. Marie Moscovici, Paris, Gallimard, 1967.

Winnicott, Donald W., The Maturational Processes and the Facilitating Environment, Londres, Karnac Books, New Ed edition, 1990.

\section{NOTES}

1. A. N. Afanassiev, Contes populaires russes, p. 141.

2. Nous nous référons ici aux idées de Carl Jung, reprises par Marie-Louise von Franz dans son analyse des contes de fées.

3. A. N. Afanassiev, « Vassilissa la belle », in op. cit., p. 140-146.

4. A. N. Afanassiev, « Tomassounet », in op. cit., p. 158-160.

5. V. I. Propp, Morphologie du conte, Paris, Gallimard, 1970.

6. J. Bellemin-Noël, Les Contes et leurs fantasmes, p. 27-29. Le plan de cet ouvrage est d'ailleurs construit sous la forme d'un menu, mettant en valeur la dimension orale des contes qu'il analyse.

7. G. Róheim, Psychoanalysis and anthropology: culture, personality and the unconscious, International Universities Press, 1968, p. 8. «Freud a interprété le fantasme du père comme animal qui mord (loup) et celui d'être mangé par son père comme la manifestation du complexe d'Oedipe négatif de passivité en relation avec le père. Mais ce fantasme est aussi une séquelle du conte de la mère cannibale qui, à son tour, nous l'avons vu, est l'enfant “cannibale" inversé. » (trad. M. Moscovici, in G. Róheim, Psychanalyse et anthropologie : Culture - Personnalité - Inconscient).

8. N. Belmont, Mythe, conte et enfance : les écritures d'Orphée et de Cendrillon, p. 133.

9. M. Klein, « Le développement précoce de la conscience chez l'enfant », p. 296-306.

10. Robert, Marthe, Roman des origines et origines du roman, Paris, Grasset, 1988.

11. Freud, Sigmund, Le roman familial du névrosé et autres textes, trad. Danièle Voldman et Olivier Mannoni, Paris, Payot, 2014. 
12. A. N. Afanassiev, «Vassilissa la belle », in op. cit., p. 142.

13. Ibid.

14. A. N. Afanassiev, « Tomassounet », in op. cit., p. 159.

15. À cet égard, cf. D. W. Winnicott, The Maturational Processes and the Facilitating Environment.

16. A. N. Afanassiev, «Vassilissa la belle », in op. cit., p. 142.

17. À cet égard, nous renvoyons à : M. Bakhtine, L'Euvre de François Rabelais et la culture populaire au Moyen Âge et sous la Renaissance.

18. A. N. Afanassiev, «Vassilissa la belle », in op. cit., p. 145.

19. N. Belmont, op. cit., p. 136.

\section{RÉSUMÉS}

La Baba Yaga est une figure complexe, appelant une démarche herméneutique plurielle. L'analyse de la problématique de la dévoration chez la sorcière dans les deux contes d'Alexandre Nikolaïevitch Afanassiev "Vassilissa la Belle » et "Tomassounet " nous amène à interroger les places que psychanalyse et littérature peuvent tenir dans l'interprétation des contes, et en quoi ces deux positions se complètent et s'enrichissent mutuellement lorsqu'il s'agit d'approcher la question de l'ambivalence de la Baba Yaga, tour à tour nourricière et sorcière.

Baba Yaga is a complex figure, and as such demands a pluralistic and open-ended hermeneutic approach. This article interrogates Baba Yaga's wish to devour as it is presented in two of Afanassiev's tales, "Vassilissa the Beautiful» and "Terechetchka». Along the way, we interrogate the role of literature and psychoanalysis in the interpretation of folk tales. These two approaches, we argue, are complementary and mutually enriching, especially when we consider the ambivalence of Baba Yaga as both a nurturing and devouring figure.

\section{INDEX}

Mots-clés: Baba Yaga, psychanalyse, littérature, ambivalence

Keywords : Baba Yaga, psychoanalysis, literature, ambivalence

\section{AUTEUR}

\section{CÉCILE ROUSSELET}

Cécile Rousselet est étudiante à l'UFR d'Études psychanalytiques de l'université Paris 7 ParisDiderot et doctorante en littérature comparée, russe et yiddish, à l'université Paris 3 SorbonneNouvelle. Elle prépare actuellement une thèse sous la direction de Carole Ksiazenicer-Matheron (Paris 3 Sorbonne-Nouvelle) et Luba Jurgenson (Paris 4 Paris-Sorbonne) : « Les personnages féminins face à l'histoire et à la mémoire dans les romans yiddish et russe après 1930 (I.J. Singer, I.B. Singer, M. Kulbak, A. Platonov, V. Grossman)». 